\title{
Investigation on Velocity Response and Energy Saving Performance of Water Hydraulic Systems Without Using Servo Valve
}

\author{
Pha N. Pham, Kazuhisa Ito, and Shigeru Ikeo* \\ Department of Machinery and Control Systems, Shibaura Institute of Technology, Saitama, Japan \\ *Department of Engineering and Applied Sciences, Sophia University, Tokyo, Japan \\ E-mail: phamngocpha@gmail.com; kazu-ito@shibaura-it.ac.jp; s_ikeo@sophia.ac.jp
}

\begin{abstract}
In recent years, water hydraulics has been getting more and more attention from the fluid power society because of its inherent merits, including environmental friendliness, high safety against fire hazards, running cost reduction, and easy availability. However, water hydraulics still has two main problems to make it popular in application; fist one is the much higher price of water hydraulic devices in comparison with the conventional one and the other is energy loss because of more leakage and larger friction than oil hydraulics due to its low viscosity. Water hydraulic systems without using servo valve such as a water hydraulic fluid switching transmission (FST) and a water hydraulic pump motor transmission (PMT) that can recover energy in deceleration period are good solutions for these difficulties.
\end{abstract}

This paper investigates two most important points on velocity response and energy saving performance consisting of recovery energy and energy consumption of water hydraulic FST and PMT. The comparison between the experimental results of these two transmissions also will be presented in this research to get the conclusion for the advantages and disadvantages of each transmission. The PMT system has proved its many advantages such as reducing noise because of smooth operation, lengthening the life duration of the devices, and the drastic reducing of both steady state error in a working phase and energy consumption. The recovered energies of the both systems are almost same and get the values from 26.3 to $31.7 \%$ of the kinetic energy of flywheel.

Keywords: Water hydraulic, fluid switching transmission, hydraulic pump motor transmission, velocity performance, energy saving, energy consumption

\section{Introduction}

In modern society, using safe and clean systems is the one of the most important requirements for technical fields; from this point, water hydraulics that uses pure tap water as pressure medium emerges as an excellent solution and it is considered as the fourth driving source beside electricity, pneumatics, and conventional oil hydraulics, especially in the fields of food and medicine processing, steel and glass production, nuclear power generation, ocean exploration, underwater robotics [i]], and underwater gait training orthosis. Water hydraulic has a very long story as mention in [2]; however, water hydraulic systems was only paid much attention by researchers from late 1990s, when advances in materials and machining technology realized the longer life times and higher flow rate and pressure for water hydraulic components [3]. Today, water hydraulics tends to divide into two branches: water hydraulic servo systems and water hydraulic systems without using servo valve.

For water hydraulic servo systems, researches mainly concen- trated on precise control such as simple adaptive control [3] and [4], robust control [5], and sliding mode control combined with disturbance observer in [6] for dealing with nonlinearities in water hydraulic component, e.g. high friction, leakage, dynamic dead zone character, and so on. The control response is getting better day by day; conventional systems using servo or proportional valves, however, remain inhere restrictions consist of expensive devices [7], especially for water hydraulics, lower energy efficiency in comparison with the system using ON/OFF valve shown in [8], and the difficulties in recovering energy in deceleration period because of conflict in control algorithm. Thus, servo valve mainly uses for the systems that require highly precise control performance and quick response.

To make water hydraulics more popular to protect environment as its original purpose, reducing cost of such systems is primary requirement. In recent years, some researches introduced hydraulic fluid switching transmission [9] or water hydraulic fluid switching transmission (FST) [U], [10], and [U]], 
that is a good method to reduce the original price of the system because of using ON/OFF valve that is much cheaper than servo valve. Moreover, FST system also can recover kinetic energy of load in deceleration period to become high pressure fluid energy stored in accumulator and it can be reused in the acceleration process in next cycle [7]. The two properties of FST system are very important and make it be useful in real application.

However, FST system inherently exposes limitations that need to be improved. First, FST system uses the fast switching logic to control the velocity; hence, the ON/OFF valves of the system switch many times in the condition of high fluid pressure that makes the hydraulic motor to change the state of working and breaking for each switching. It causes unendurable noise and the duration of the motor to be reduced. Second, to control the velocity of the load, it needs setting a velocity threshold to make the velocity theoretically work inside the bounds, but in fact, the steady state error goes beyond the bounds because of the time delay of devices. The methods to reduce the error are using the quick response devices and the restricting the velocity threshold as mentioned in [ [ $]$ ] and [II]]; however, the restriction has its limit depending on the characteristic of the ON/OFF valves, that means the steady state error inherently remains in the control logic. The first method will bring the price of the system up and the second one will make the number of switching of ON/OFF valves increase, that leads to shorten the lifetime of the valves. Last, in working phase, FST system operates in condition of high pressure that acts as the load of hydraulic pump; thus, the system dissipates large amounts of energy, because an amount not less losses via relief valves and other amount stores in shock absorbed accumulator and then disappears after action cycle.

To deal with the limitations of FST system, this research studied on a new water hydraulic transmission that is the directly connection of a water hydraulic pump and a motor, it can be called a water hydraulic pump motor transmission (PMT). In the past, there are very few research concerned with the system that is the direct connection between a hydraulic pump and an actuator such as in [12] and [13]; however, all the researches worked for controlling the displacement of a cylinder and the systems cannot recover energy in the deceleration period. PMT system mentioned in this paper is an improvement of FST system. It can work smoother and reduce noise drastically because ON/OFF valves only switch one time in all cycle consist of acceleration, working or constant, and deceleration phases. By this way, the durations of the hydraulic motor and ON/OFF valves will be lengthened due to stable working pressure and much fewer states changing.

The main objectives of this study are twofold:

1. To examine the velocity responses of FST and PMT systems and then compare the results to prove the advantage of PMT in experiments.

2. To evaluate the saved energies of FST and PMT systems based on energy consumption in all a working cycle and recovered energy in deceleration period. From these results, compare the consumption of each system.
The remainder of the paper is organized as follows. In Section 2, the introductions of FST and PMT systems including the structures, operations, and specifications of experimental devices are shown. Then, the control logics of FST and PMT system are presented in Section 3. Sections 4 and 5 are the experimental results of velocity responses and energy saving for FST and PMT systems, and at the end of each section is the comparison of results of FST and PMT system to draw conclusions.

\section{FST and PMT Systems}

In this section, the structures, operations, and specification of experimental devices of the two water hydraulic transmissions, FST and PMT, will be presented and discussed.

\subsection{FST System}

A simple FST circuit used for experiments in this research will be introduced in fig. I. The system consists of following main elements: a fixed displacement pump (P) that is connected with an electric motor $(\mathrm{M})$, a fixed displacement pump/motor (PM), two accumulators $\left(\mathrm{ACC}_{i}, i=1,2\right)$, three ON/OFF valves $\left(\mathrm{VS}_{i}, i=1, \ldots, 3\right)$. The two accumulators $\mathrm{ACC}_{1}$ and $\mathrm{ACC}_{2}$ are used as a pressure surge absorber and an energy storage, respectively. The fluid energy with the pressure $p_{s}$ generated by the pump $\mathrm{P}$ is considered as the input energy, kept as $12 \mathrm{MPa}$ in all experiments. The flywheel (FW) connected to PM is considered as a working load. The specifications of main experimental devices will be shown in tab. ‥

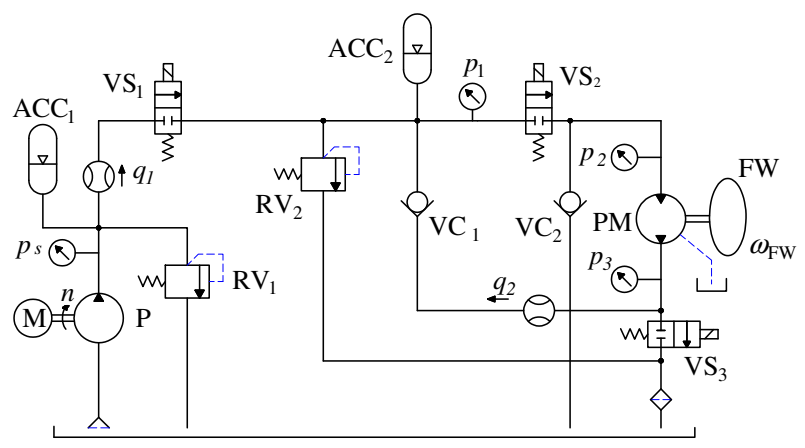

Figure 1: Schematic of water hydraulic FST system.

The goal is to control the flywheel velocity to meet the desired constant velocity at the working time. An operational cycle can be divided into three phases: an acceleration phase, a working or constant phase, and a deceleration phase as shown in fig. 4 (a). In the acceleration phase, there is no control for ON/OFF valves, they are opened to make the water flow directly from the pump $\mathrm{P}$ to the pump/motor PM. After once getting the desired constant velocity, the state of the system is changed to the working phase. At this time, the control logics for ON/OFF valves are used to force the flywheel velocity to oscillate around the constant value. When the working finishes, the system turns to the deceleration phase, the electric motor $\mathrm{M}$ is stopped and all the ON/OFF valves are in close state; however, the pump/motor PM still works because 
Table 1: Specifications of experimental devices.

\begin{tabular}{lll}
\hline \multicolumn{1}{c}{ Symbols } & \multicolumn{1}{c}{ Specifications } & \multicolumn{1}{c}{ Values } \\
\hline $\mathrm{P}$ & Displacement volume & $15 \times 10^{-6} \mathrm{~m}^{3}$ \\
$\mathrm{PM}$ & Displacement volume & $15 \times 10^{-6} \mathrm{~m}^{3}$ \\
$\mathrm{ACC}_{1}$ & $\mathrm{~N}_{2}$ gas volume & $0.005 \mathrm{~m}^{3}$ \\
& Precharge pressure & $5.0 \mathrm{MPa}$ \\
$\mathrm{ACC}_{2}$ & $\mathrm{~N}_{2}$ gas volume & $0.01 \mathrm{~m}^{3}$ \\
& Precharge pressure & $7.8 \mathrm{MPa}$ \\
$\mathrm{FW}$ & Moment of inertia & $1.58 \mathrm{kgm}$ \\
& Mass & $78.9 \mathrm{~kg}$ \\
& Diameter & $0.4 \mathrm{~m}$ \\
& Thickness & $0.08 \mathrm{~m}$ \\
Electric motor & Revolution & $1200 \mathrm{~min}^{-1}$ \\
\hline
\end{tabular}

of kinetic energy of flywheel and it acts as a hydraulic pump to convert the kinetic energy into high pressure fluid that is stored in the accumulator $\mathrm{ACC}_{2}$, it supplies the water via the suction line that contains the check valve $\mathrm{VC}_{2}$. This energy is called recovery energy and will be reused in the next action cycle in the acceleration phase to make the transient time of the acceleration phase shorten, and also to reduce the energy consumption in this cycle.

\subsection{PMT System}

A new water hydraulic pump motor transmission PMT will be introduced in this subsection, fig. $\square$ is the schematic of the system. The PMT system is set on the basis of the FST system, only some devices was changed and the control algorithms for the ON/OFF valves and the electric motor $\mathrm{M}$ are different. Note that, the same symbols of devices of the FST and PMT systems in figs. $\square$ and $\square$ represent for the same devices in the real systems, and the specifications of the experimental devices of the PMT system can be seen in the tab. In the PMT system, the ON/OFF valve $\mathrm{VS}_{1}$ was replaced by the check valve $\mathrm{VC}_{3}$, the accumulator $\mathrm{ACC}_{1}$ was eliminated, and the accumulator $\mathrm{ACC}_{2}$ was used for both tasks: pressure surge absorber in acceleration and constant phases and energy storage for recovered energy in deceleration phase.

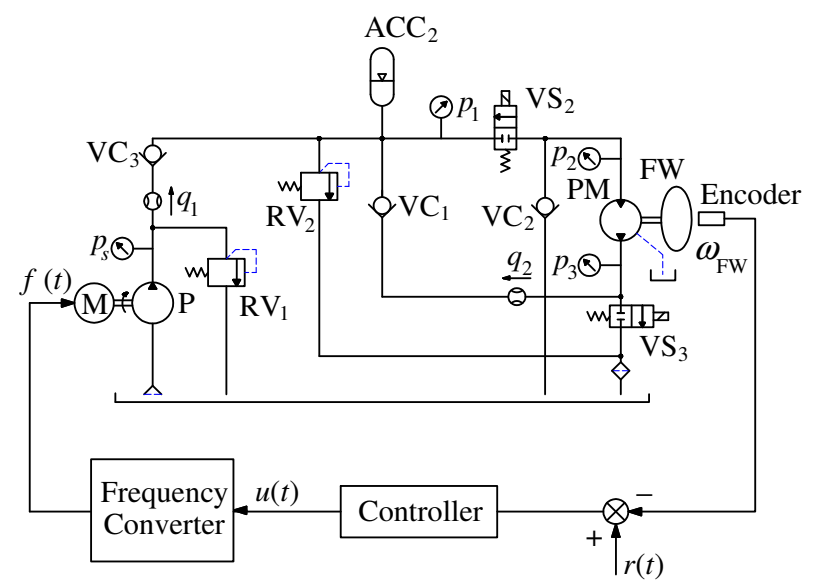

Figure 2: Schematic of water hydraulic PMT system.
The most important point of the PMT system is control logic for the two ON/OFF valves $\mathrm{VS}_{2}, \mathrm{VS}_{3}$, and the electric motor $\mathrm{M}$. In the acceleration and constant phases, all the two $\mathrm{ON} / \mathrm{OFF}$ valves $\mathrm{VS}_{2}$ and $\mathrm{VS}_{3}$ are opened and they will be closed for all time of the deceleration phase. The controller receives the reference signal $r(t)$ as in fig. $\square$ and the feedback of the flywheel velocity that is provided by an encoder to generate the control signal $u(t)$. The hardware for controller is $\mathrm{dSPACE}$ and the software is MATLAB; thus the control signal $u(t)$ is limited by \pm 10 Voltage. The rotational velocity of an electric motor can be calculated via following equation [114]

$$
n=120 \frac{f}{P}
$$

where $n$ is the rotational velocity of the electric motor, $f$ the frequency of electrical power supply, and $P$ the number of poles of the motor, in this case $P=4$. Thus, the control signal that is dilivered to the electric motor $\mathrm{M}$ is frequency. A frequency converter is used for converting the control signal out of dSPACE in voltage to frequency signal supplied to the electric motor $\mathrm{M}$. The specification of the frequency converter can be derived by doing some tests on it. The experimental

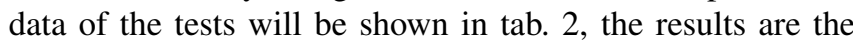
average of five times of experiments; for more detail, fig. [3] shows the input-output mapping of the frequency converter.

Table 2: Test data of the frequency converter.

\begin{tabular}{cc}
\hline Input $(\mathrm{V})$ & Output $(\mathrm{Hz})$ \\
\hline 0.1 & 0.70 \\
0.2 & 1.30 \\
0.4 & 2.60 \\
0.8 & 5.08 \\
1.0 & 6.30 \\
1.5 & 9.40 \\
2.0 & 12.50 \\
2.5 & 15.50 \\
3.0 & 18.70 \\
3.5 & 21.75 \\
4.0 & 24.80 \\
\hline
\end{tabular}

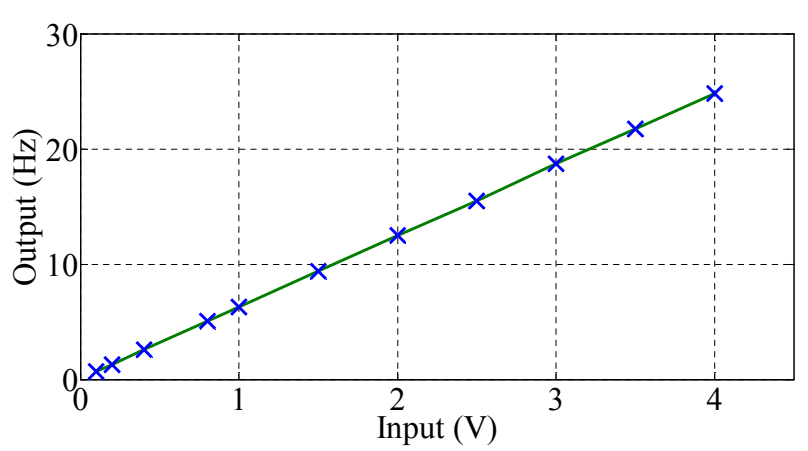

Figure 3: Frequency converter input-output mapping.

From the experimental results, it is easy to realize that, the relationship of the input and output of the frequency converter 
can be considered as linear mapping, and it can be written approximately as following equation

$$
f=\varepsilon u,
$$

where $\varepsilon$ is the frequency coefficient and get the value of $6.1817 \mathrm{~Hz} / \mathrm{V}$.

In the deceleration phase, all the ON/OFF valves are closed as mentioned above, and the electric motor $\mathrm{M}$ are also stopped. Thus, there is no energy supply to the hydraulic pump/motor PM but it still works same as FST system and also converts the kinetic energy of the flywheel FW into high pressure fluid stored in the accumulator $\mathrm{ACC}_{2}$.

\section{Control Logics of FST and PMT Systems}

\subsection{FST System}

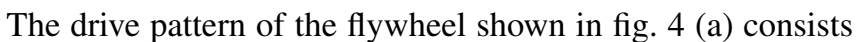
of three simple phases: an acceleration phase (Phase 1), a constant speed phase (Phase 2), and a deceleration phase (Phase 3). This section will explain the logic to control the ON/OFF valves, depending on the flywheel drive pattern and the variable reference velocity of the flywheel (fig. 因(b)). The valve $\mathrm{VS}_{1}$ is opened in both Phase 1 and Phase 2 , and only closed in Phase 3. The two valves $\mathrm{VS}_{2}$ and $\mathrm{VS}_{3}$ received the same control signal depending on the flywheel rotational velocity $\omega_{\mathrm{FW}}$. The electric motor $\mathrm{M}$ is operated with the constant velocity in all Phases 1 and 2, it is stopped in Phase 3 for saving energy. Following, the valve switching algorithms corresponding to the three phases will be introduced.

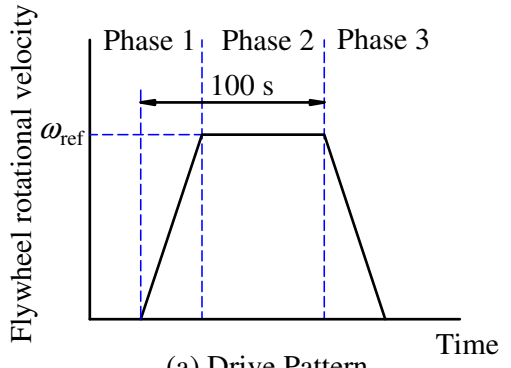

(a) Drive Pattern

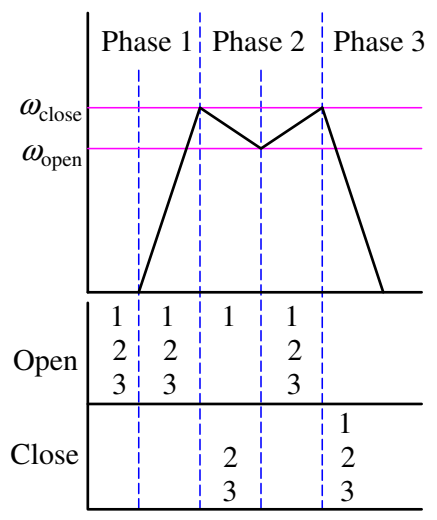

(b) Valve control logics

Figure 4: Control logic of ON/OFF valves.

\subsubsection{Acceleration Phase (Phase 1)}

In this phase, the FST accelerates the flywheel FW from stationary state to a given reference rotational velocity. Once reached to the reference rotational velocity, the control system is switched to Phase 2. In this phase, all of the ON/OFF valves are opened full time and the electric motor is operated with the velocity of $1200 \mathrm{~min}^{-1}$.

\subsubsection{Constant (Working) Phase (Phase 2)}

This phase maintains the rotational velocity of the flywheel FW between $\omega_{\text {open }}$ and $\omega_{\text {close }}$ as in fig. $⿴ 囗 十$ (b). Two Phases 1 and 2 take 100 seconds. Tables 3 presents the control logics of the three ON/OFF valves. In the control logic, when the velocity of flywheel reaches out of $\omega_{\text {close }}$, the both ON/OFF valves $\mathrm{VS}_{2}$ and $\mathrm{VS}_{3}$ are changed the state from open to close to brake the flywheel. Inversely, when the velocity downs less than $\omega_{\text {open }}$, the state of the both ON/OFF valves $\mathrm{VS}_{2}$ and $\mathrm{VS}_{3}$ are altered from close to open. By this control logic, the velocity of the flywheel will oscillate theoretically inside $\omega_{\text {open }}$ and $\omega_{\text {close }}$; however, in fact, the steady state error of the flywheel velocity is larger because of the effect of the time delay of the real devices in the system.

Table 3: Valve operation logic in Phase 2.

\begin{tabular}{ccc}
\hline Valves & Open & Close \\
\hline $\mathrm{VS}_{1}$ & \multicolumn{2}{c}{ Open } \\
$\mathrm{VS}_{2}$ & $\omega_{\mathrm{FW}}<795 \mathrm{~min}^{-1}$ & $\omega_{\mathrm{FW}}>805 \mathrm{~min}^{-1}$ \\
$\mathrm{VS}_{3}$ & $\omega_{\mathrm{FW}}<795 \mathrm{~min}^{-1}$ & $\omega_{\mathrm{FW}}>805 \mathrm{~min}^{-1}$ \\
\hline
\end{tabular}

\subsubsection{Deceleration Phase (Phase 3)}

In the final phase, the speed of the flywheel is decelerated to zero and note that also in this phase, no velocity control is executed. In this phase, all the ON/OFF valves are closed, the closing of ON/OFF valve $\mathrm{VS}_{3}$ is to brake the flywheel FW and also induce energy recovery process, ON/OFF valve $\mathrm{VS}_{1}$ is closed to cut the supply energy, and ON/OFF valve $\mathrm{VS}_{2}$ is closed to keep the recovery energy inside the accumulator $\mathrm{ACC}_{2}$. The recovery energy is mainly produced in this Phase; at this time, the pump/motor PM is act as a pump to convert kinetic energy of the flywheel into high pressure fluid that is stored in the accumulator $\mathrm{ACC}_{2}$.

\subsection{PMT System}

The controller of PMT system that is depicted in fig. \$ is a feedback control that aims to make the velocity of the flywheel track a desired reference velocity $r(t)$ as shown in fig. 2 . The controller in this study is conventional PID control that was tuned manually to attenuate the steady state error and overshoot. The PID controller has the transfer function

$$
G_{c}(s)=K_{P}+\frac{K_{I}}{s}+K_{D} s,
$$

where $K_{P}, K_{I}$, and $K_{D}$ are the proportional, integral, and derivative gains; in the experiment, they get the value of 0.002 , 


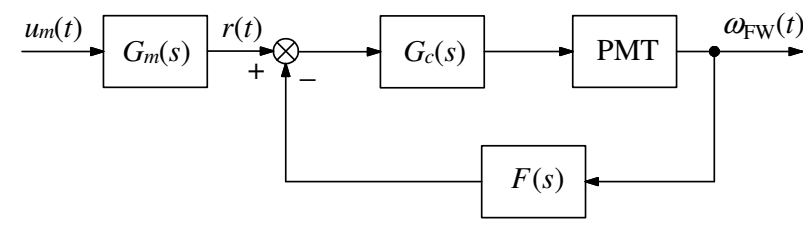

Figure 5: Control structure of PMT system.

0.001, and 0.001, respectively.

In the feedback loop, a first order low-pass filter that can be described by eq. (四) was used to eliminate the high frequency noise caused by measurement devices that consist of an encoder and a frequency-velocity (FV) converter.

$$
F(s)=\frac{10}{s+10}
$$

The reference $r(t)$ was generated after a reference model $G_{m}(s)$ as in eq. (5) by a rectangular input signal $u_{m}(t)$.

$$
G_{m}(s)=\frac{1}{2 s+1} .
$$

\section{Velocity Response}

In this section, the velocity response of the flywheel FW for both FST and PMT systems will be shown and compared.

\subsection{FST System}

In the drive pattern as shown in fig. [3 (a) consists of three phases; however, Phase 2 is the most important for velocity response because the system will work and do its function mainly in this phase. Phase 2 is also called constant (velocity) phase or working phase and its reference velocity $\omega_{w r}$ in this research is constant for the values from $600 \mathrm{~min}^{-1}$ to $1000 \mathrm{~min}^{-1}$. Table $耳$ is bounds of the velocity responses at Phase 2 of FST system when reference velocity $\omega_{w r}$ varies from $600 \mathrm{~min}^{-1}$ to $1000 \mathrm{~min}^{-1}$ and fig. 6 has a more detail view about the velocity response when the reference velocity $\omega_{w r}$ is $800 \mathrm{~min}^{-1}$.

Table 4: Experimental velocity response of FST system.

\begin{tabular}{ccccc}
\hline $\begin{array}{c}\text { Reference velocity } \\
\omega_{w r}\left[\mathrm{~min}^{-1}\right]\end{array}$ & $\begin{array}{c}\omega_{\min } \\
{\left[\mathrm{min}^{-1}\right]}\end{array}$ & $\begin{array}{c}\omega_{\max } \\
{\left[\mathrm{min}^{-1}\right]}\end{array}$ & $\begin{array}{c}e_{\min } \\
{[\%]}\end{array}$ & $\begin{array}{c}e_{\max } \\
{[\%]}\end{array}$ \\
\hline 1000 & 968 & 1028 & 3.20 & 2.80 \\
900 & 869 & 927 & 3.44 & 3.00 \\
800 & 767 & 827 & 4.13 & 3.38 \\
700 & 669 & 726 & 4.43 & 3.71 \\
600 & 570 & 625 & 5.00 & 4.17 \\
\hline
\end{tabular}

As showing in tab. 团 the flywheel velocity errors was regulated in a range from $-33 \mathrm{~min}^{-1}$ to $28 \mathrm{~min}^{-1}$ corresponding with the bound of percentage errors $e_{\min }$ and $e_{\max }$ that will be defined in eqs. 6 and $\square$ to $5 \%$ and $4.17 \%$, respectively, when the reference velocity changes from $600 \mathrm{~min}^{-1}$ to $1000 \mathrm{~min}^{-1}$. Note that these bounds of the flywheel velocity errors are almost same when the reference velocity varies,

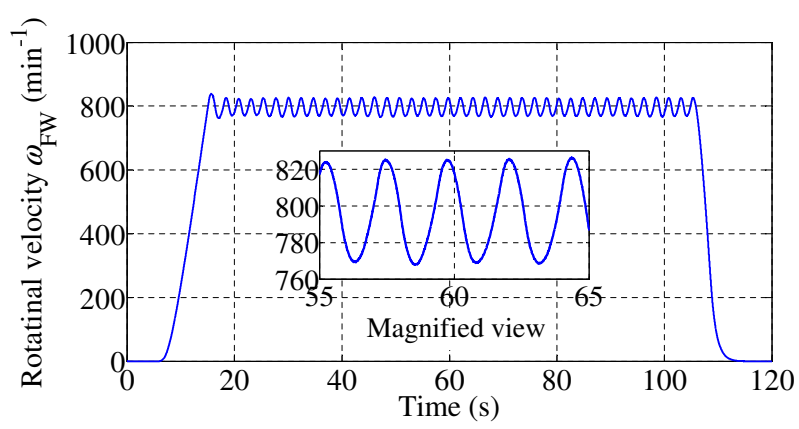

Figure 6: Experimental velocity response of FST system $\left(\omega_{\mathrm{wr}}=800 \mathrm{~min}^{-1}\right)$.

they depend mainly in the range of the threshold and the time delay of the devices as mentioned in [ [ 7$]$,

$$
\begin{aligned}
& e_{\min }=\frac{\omega_{w r}-\omega_{\min }}{\omega_{\min }}, \\
& e_{\max }=\frac{\omega_{\max }-\omega_{w r}}{\omega_{\max }},
\end{aligned}
$$

where $\omega_{\min }$ and $\omega_{\max }$ are the minimum and maximum velocity in the working phase.

Another property of the flywheel velocity response in Phase 2 needs to be noticed is that the velocity response of FST system oscillates around the reference velocity. Figure $\square$ shows the control signal of the ON/OFF valves $\mathrm{VS}_{2}$ and $\mathrm{VS}_{3}$ that presents for the $\mathrm{ON}$ or OFF state of the valves. With the working time of 100 seconds, the number of switching of the valves is 80 times. The oscillation of the velocity is not good for operation of the system, so it should be improved. The excessive changing states of the ON/OFF valves make the lifetime of not only the ON/OFF valves but also the hydraulic pump/motor PM decrease.

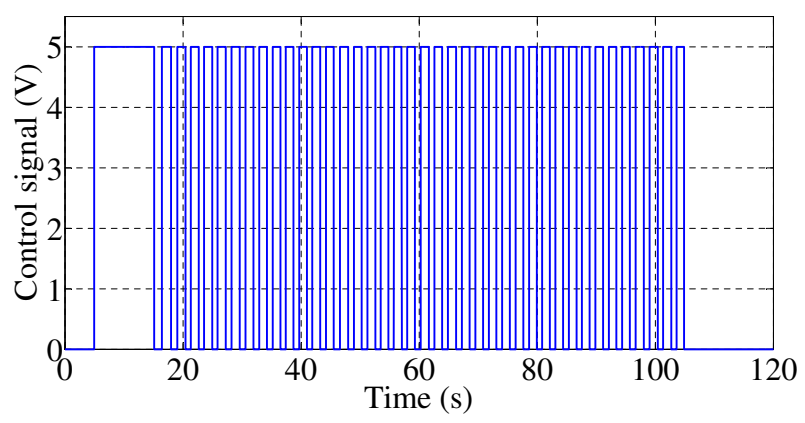

Figure 7: Control signal of ON/OFF valves $V S_{2}$ and $V S_{3}$.

\subsection{PMT System}

PMT system was controlled by a conventional PID as mentioned above, the target of the control is to make the velocity of the flywheel converge to a reference signal $r(t)$. To compare with the velocity response of FST, the reference signal is also divided into three parts: an acceleration phase, a constant (working ) phase, and a deceleration phase. The acceleration phase can be calculated when the velocity is the stationary state to get the constant value of the reference signal $\omega_{w r}$, the 
constant and working phase is defined same as FST system, the acceleration and constant phases also took 100 seconds for each cycle.

The velocity responses in working phase of PMT system corresponding with the reference velocity $\omega_{w r}$ from $600 \mathrm{~min}^{-1}$ to $1000 \mathrm{~min}^{-1}$ are shown in tab. [5, and fig. $\mathbb{8}$ shows the velocity response in case of reference velocity $\omega_{w r}=800$ $\min ^{-1}$. Base on the tab. 5, it is easy to realized that the limit of the bounds of the velocity errors is $3.92 \mathrm{~min}^{-1}$ and $4.18 \mathrm{~min}^{-1}$ corresponding with the bound of percentage errors $e_{\min }$ and $e_{\max }$ that also will be defined in eqs. 6 and $\square$ to $0.49 \%$ and $0.52 \%$, respectively, when the reference velocity changes from $600 \mathrm{~min}^{-1}$ to $1000 \mathrm{~min}^{-1}$. As showed in fig. प, the transient time (in the acceleration phase) of the response takes longer time than the reference, it can be improved by using larger gains of PID controller, however, it will bring the larger overshoot. Thus, finding a method to reduce the transient time and still keep a small overshoot is a requirement of this research in the future.

Table 5: Experimental velocity response of PMT system.

\begin{tabular}{ccccc}
\hline $\begin{array}{c}\text { Reference velocity } \\
\omega_{w r}\left[\mathrm{~min}^{-1}\right]\end{array}$ & $\begin{array}{c}\omega_{\min } \\
{\left[\mathrm{min}^{-1}\right]}\end{array}$ & $\begin{array}{c}\omega_{\max } \\
{\left[\min ^{-1}\right]}\end{array}$ & $\begin{array}{c}e_{\min } \\
{[\%]}\end{array}$ & $\begin{array}{c}e_{\max } \\
{[\%]}\end{array}$ \\
\hline 1000 & 996.85 & 1003.20 & 0.32 & 0.32 \\
900 & 896.88 & 904.18 & 0.35 & 0.46 \\
800 & 796.08 & 802.99 & 0.49 & 0.37 \\
700 & 697.22 & 704.10 & 0.40 & 0.59 \\
600 & 598.34 & 603.13 & 0.28 & 0.52 \\
\hline
\end{tabular}

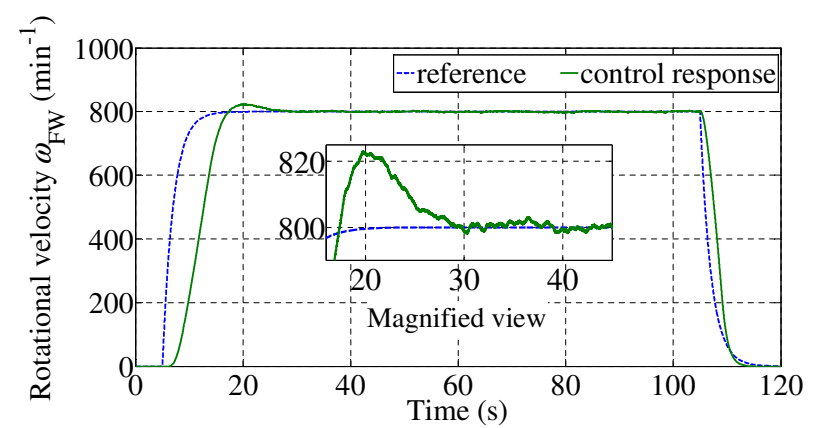

Figure 8: Velocity response of PMT system $\left(\omega_{\mathrm{wr}}=800\right.$ $\left.\min ^{-1}\right)$.

Figure 9 shows the control signal of the electric motor M. In the working phase, the controller sent to the motor a signal that is not changed much. This is really good for the duration of the motor. For all the time of the acceleration and working phases, the ON/OFF valve $\mathrm{VS}_{2}$ and $\mathrm{VS}_{3}$ were opened, that made the supply pressure for hydraulic pump/motor PM was steady; therefore, the lifetime of the both ON/OFF valves and also the hydraulic pump/motor is not affected.

\subsection{Comparison}

This subsection aims to analyse and compare the velocity response between FST and PMT to derive the advantage and disadvantage of each transmission. The percentage errors $e_{\min }$

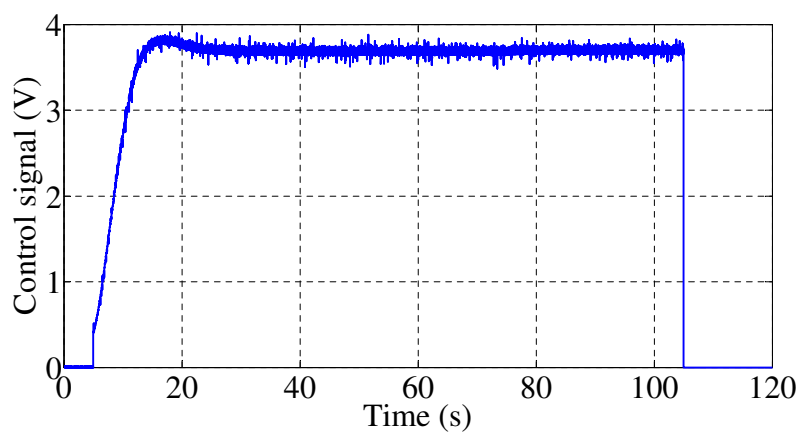

Figure 9: Control signal of electric motor.

and $e_{\max }$ are shown in figs. 10 and 10 , respectively. Base on the figures and tabs. 4 and 5 , it can be concluded that the steady state error of PMT system is much smaller than FST system in both percentage errors $e_{\min }$ and $e_{\max }$ and the maximum difference is nearly 18 times and the minimum one is approximately 6 times.

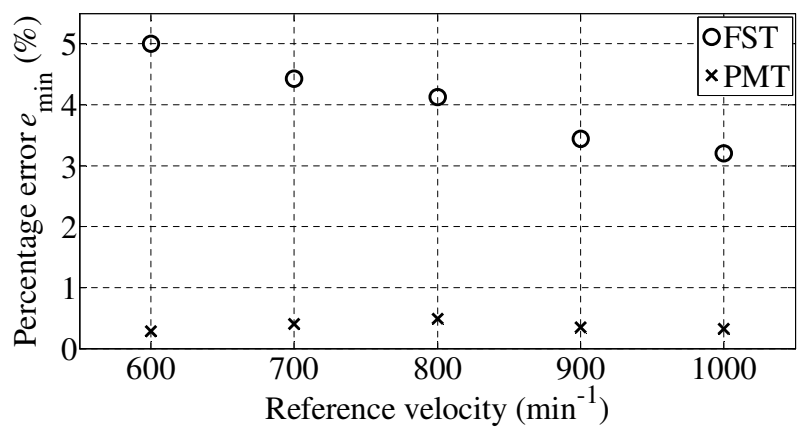

Figure 10: Percentage error $e_{\min }$.

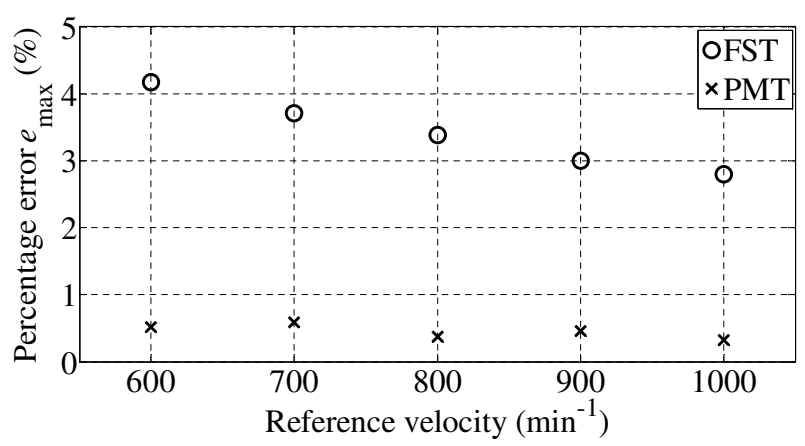

Figure 11: Percentage error $e_{\max }$.

Figure [2] shows the velocity responses of both FST and PMT systems in case of reference velocity $\omega_{w r}=800 \mathrm{~min}^{-1}$. At the beginning of the acceleration phase, the control performances of the both transmissions are almost same; only the end of this phase, the PMT responded slower than FST a little bit however it is smoother. In the most important phase, working phase, the flywheel velocity of PMT system not only much more precise but also is smoother and does not oscillate rapidly in comparison with FST system. 


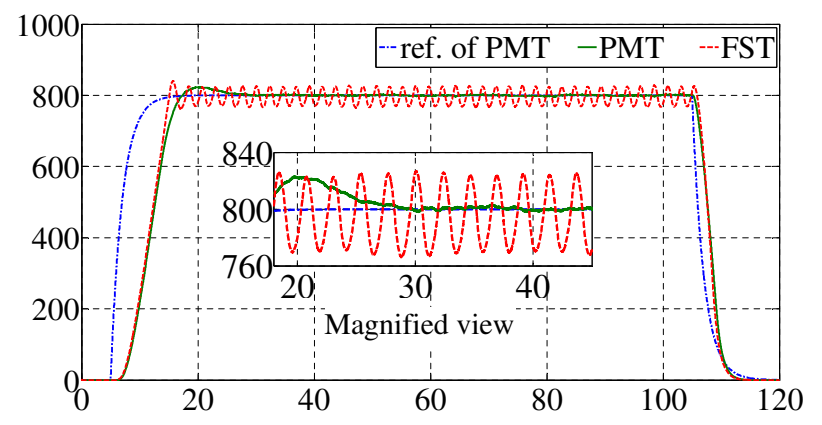

Figure 12: Velocity responses of FST and PMT $\left(\omega_{w r}=\right.$ $800 \min ^{-1}$ ).

\section{Energy Saving}

Energy saving is one of most important property of water hydraulic systems without using servo valve. The energy saving of both FST and PMT systems that concerns with recovered energy in deceleration process and energy consumption in acceleration and working phase will be presented in this section.

\subsection{Energy Consumption}

Because of the energy loss of the water hydraulic transmissions is considerably higher than conventional one; thus, taking into account of the energy consumption is an important requirement of the water hydraulic systems in general and the water hydraulic systems without using servo valve in particular.

\subsubsection{FST system}

Energy consumption of the FST system is only for Phase 1 and Phase 2, because in Phase 3, the electric motor $M$ is stopped. Table 6 shows the energy consumptions of the system in a cycle when the reference velocity varies from $600 \mathrm{~min}^{-1}$ to $1000 \mathrm{~min}^{-1}$. The energy consumptions are almost same and decrease if the reference velocity increases. The reason for this can be explained as follows. The velocity of the electric motor $\mathrm{M}$ is constant for all the reference velocities and gets the value of approximately $1200 \mathrm{~min}^{-1}$ to force the system to complete full cycle when the reference velocity gets the highest value of $1000 \mathrm{~min}^{-1}$. The energy consumption for the acceleration phase is higher if the reference velocity is bigger; however, in phase 2 , when the reference velocity is bigger, the flow rate flows through the hydraulic pump/motor is also bigger and the supply flow rate from the hydraulic pump $\mathrm{P}$ is almost constant, it leads to the working pressure that can be considered as load of the hydraulic motor is smaller and the energy consumption of the electric motor will decrease in this phase. The energy consumption is the combination of the both phases. Thus the energy consumption of the system depends on both the working time of the system and the reference velocity.

\subsubsection{PMT system}

Energy consumption of PMT system is only for the acceleration and working phases same as FST system, because the electric motor $\mathrm{M}$ is also stopped in the deceleration phase.
Table 6: Energy consumption of FST system.

\begin{tabular}{cc}
\hline $\begin{array}{c}\text { Reference velocity } \\
\omega_{w r}\left[\mathrm{~min}^{-1}\right]\end{array}$ & $\begin{array}{c}\text { Energy consumption } \\
{[\mathrm{Wh}]}\end{array}$ \\
\hline 1000 & 137 \\
900 & 138 \\
800 & 140 \\
700 & 140 \\
600 & 141 \\
\hline
\end{tabular}

Energy consumption of the system when the reference velocity is from $600 \mathrm{~min}^{-1}$ to $1000 \mathrm{~min}^{-1}$ will be shown in tab. $\square$. The energy consumptions of PMT system decreases if the reference velocity goes down. It is not difficult to understand because the velocity of the electric motor $\mathrm{M}$ also varies depending on the reference velocity.

Table 7: Energy consumption of PMT system.

\begin{tabular}{cc}
\hline $\begin{array}{c}\text { Reference velocity } \\
\omega_{w r}\left[\mathrm{~min}^{-1}\right]\end{array}$ & $\begin{array}{c}\text { Energy consumption } \\
{[\mathrm{Wh}]}\end{array}$ \\
\hline 1000 & 28.5 \\
900 & 26.0 \\
800 & 23.0 \\
700 & 20.8 \\
600 & 18.4 \\
\hline
\end{tabular}

\subsubsection{Comparison}

The comparison of the energy consumption between FST and PMT is one of the major purposes of this research. Figure 13 shows the energy consumption of FST and PMT systems. Based on the figure and tabs. 6 and Z, the advantage in the energy saving of PMT is displayed clearly. The minimum difference between FST and PMT is 4.8 times and the maximum one is 7.7 times corresponding with the reference velocities of 1000 $\min ^{-1}$ and $600 \mathrm{~min}^{-1}$, respectively. The difference is because of the energy loss of FST system via relief valve, stored in $\mathrm{ACC}_{1}$ that will disappear after the cycle. Morover, the energy lose when the ON/OFF valves change its state that contributes the bigger part and the energy stored in accumulator $\mathrm{ACC}_{2}$ which can be reused same as the recovered energy.

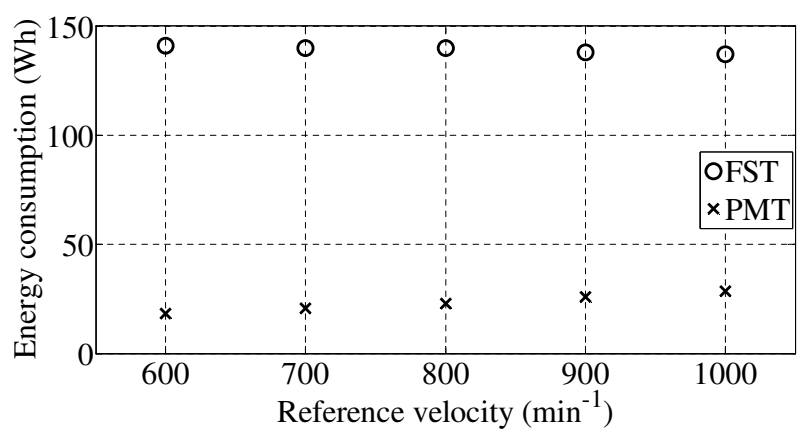

Figure 13: Energy consumption of FST and PMT. 


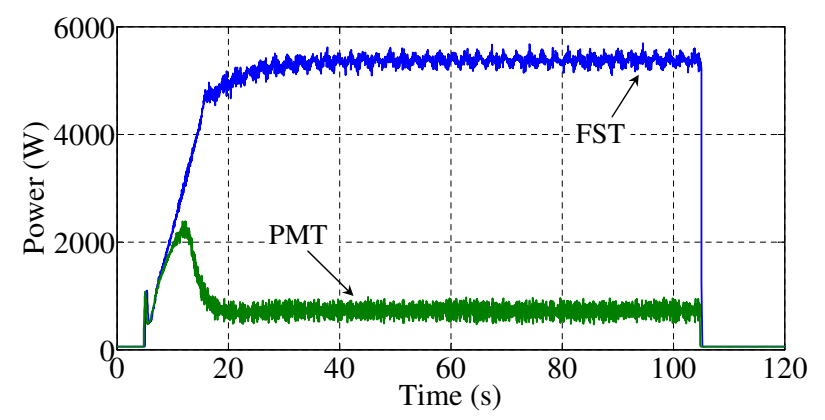

Figure 14: Electric motor supply power of FST and PMT $\left(\omega_{w r}=800 \min ^{-1}\right)$.

Figure 144 shows the instantaneous value of the supply power to the electric motor M of FST and PMT system in experiment. At the beginning of the acceleration phase, the energy consumption of FST and PMT is similar; however, at the end of the phase, energy consumption of FST still increase and the energy consumption of PMT reduces, because the velocity of the electric motor M increases rapidly for FST case and slower for PMT case that make the velocity response of PMT is slower. In the working phase, the energy consumption of FST is much higher than PMT because the pressure in the output line of the hydraulic motor of FST system is higher than PMT system.

\subsection{Energy Recovery Efficiency}

Energy recoveries of both FST and PMT systems were produced in the deceleration phase. The deceleration phases of FST and PMT systems were exactly same, all ON/OFF valves were closed, the electric motor $M$ was stopped. ON/OFF valve $\mathrm{VS}_{3}$ was closed to break the flywheel; at that time, the hydraulic pump motor PM acted as a pump and converted the kinetic energy of the flywheel FW that caused by high speed of the flywheel into high pressure water and it was stored in the accumulator $\mathrm{ACC}_{2}$. The recovered energy would be reused in the next cycle of the systems. The ON/OFF switching valve $\mathrm{VS}_{2}$ was closed to cut the supply energy to the hydraulic pump/motor PM directly and preserve the recovered energy. The recovered energy stored in the accumulator $\mathrm{ACC}_{2}$ is defined as follows

$$
E_{\text {recovery }}=\int_{t_{\text {start3 }}}^{t_{\text {end3 }}} p_{\mathrm{ACC}_{2}} \cdot q_{\mathrm{ACC}_{2}} d t
$$

where $E_{\text {recovery }}$ is the recovered energy to the accumulator $\mathrm{ACC}_{2}$ during the deceleration phase, $p_{\mathrm{ACC}_{2}}$ the pressure of the accumulator $\mathrm{ACC}_{2}, q_{\mathrm{ACC}_{2}}$ the flow rate stored in the accumulator $\mathrm{ACC}_{2}$, and $t_{\mathrm{star} 3}, t_{\mathrm{end} 3}$ the times at the beginning (the time of 105 second) and the end of Phase 3, respectively. Because the pressure $p_{\mathrm{ACC}_{2}}$ and the flow rate $q_{\mathrm{ACC}_{2}}$ are same as the pressure $p_{1}$ and the flow rate $q_{2}$, eq. (Q) can be written as

$$
E_{\text {recovery }}=\int_{t_{\text {start3 }}}^{t_{\text {end3 } 3}} p_{1} \cdot q_{2} d t .
$$

To estimate the recovered energy, the efficiency index $\eta$ will be introduced as the ratio of the recovered energy and the maximum kinetic energy of the flywheel FW in the deceleration phase as follows

$$
\eta=\frac{E_{\text {recovery }}}{E_{\mathrm{FW}}}
$$

where $E_{\mathrm{FW}}$ is the maximum kinetic energy possessed by the flywheel in Phase 3. This evaluates how much energy will be recovered from the kinetic energy of the flywheel dissipated in conventional operations and can be reused in next action cycle in real application. In this case, $E_{\mathrm{FW}}$ is calculated by following equation

$$
E_{\mathrm{FW}}=\frac{1}{2} I \omega_{\max }^{2}
$$

where $\omega_{\max }$ is the maximum rotational velocity of the flywheel in the deceleration phase.

Table 8: Recovered energy of FST system.

\begin{tabular}{cccc}
\hline $\begin{array}{c}\text { Reference velocity } \\
\omega_{w r}\left[\mathrm{~min}^{-1}\right]\end{array}$ & $\begin{array}{c}E_{\text {recovery }} \\
{[\mathrm{kJ}]}\end{array}$ & $\begin{array}{c}E_{\mathrm{FW}} \\
{[\mathrm{kJ}]}\end{array}$ & $\begin{array}{c}\eta \\
{[\%]}\end{array}$ \\
\hline 1000 & 3.17 & 8.65 & 36.7 \\
900 & 2.49 & 7.16 & 34.8 \\
800 & 1.88 & 5.67 & 33.2 \\
700 & 1.27 & 4.22 & 30.1 \\
600 & 0.86 & 3.27 & 26.3 \\
\hline
\end{tabular}

Table 9: Recovered energy of PMT system.

\begin{tabular}{cccc}
\hline $\begin{array}{c}\text { Reference velocity } \\
\omega_{w r}\left[\mathrm{~min}^{-1}\right]\end{array}$ & $\begin{array}{c}E_{\text {recovery }} \\
{[\mathrm{kJ}]}\end{array}$ & $\begin{array}{c}E_{\mathrm{FW}} \\
{[\mathrm{kJ}]}\end{array}$ & $\begin{array}{c}\eta \\
{[\%]}\end{array}$ \\
\hline 1000 & 3.21 & 8.66 & 37.1 \\
900 & 2.48 & 7.02 & 35.3 \\
800 & 1.85 & 5.54 & 33.4 \\
700 & 1.29 & 4.25 & 30.4 \\
600 & 0.83 & 3.12 & 26.6 \\
\hline
\end{tabular}

Tables $\nabla$ and $Q$ shows the recovered energies of FST and PMT systems in the deceleration phase, all the results are the average of five times experiments. From the tables, it is easy to realize that the recovered energy of the FST and PMT systems are almost same; however, they still exist a minor difference because the maximum rotational velocities of the flywheel in the deceleration phase $\omega_{\max }$ of the FST and PMT systems are not the same and the measurement devices have its deviations. The recovered energies of the both systems increased when the reference velocities raised and got the maximum values of 3.17 and 3.21 corresponding with the energy recovery efficiency index $\eta$ of $36.7 \%$ and $37.1 \%$ for the FST and PMT systems, respectively.

\section{Conclusion}

This paper is an investiagation on the FST and PMT systems that do not use servo valve. The easiest advantage of the systems is cheap and the energy loss can be reduced. 
The FST system is good at recovering energy in the deceleration period, it is almost same as the PMT system, and it can save from 26.3 to $26.7 \%$ of the kinetic energy of the flywheel. However, the steady state error of the flywheel velocity in the working period is still large, it is from 2.8 to $5 \%$ and it is very difficult to be reduced because the error exists in the control logic. The energy consumption of the system is quite high because of lossing via the relief valves, the accumulator $\mathrm{ACC}_{1}$, and the process of changing state of ON/OFF valves. It needs from 137 to $141 \mathrm{Wh}$ for all acceleration and working phases that last 100 seconds when the reference velocity changes from 600 to $1000 \mathrm{~min}^{-1}$.

The PMT system displayed more advantage than FST system. Fist, the system can reduce the noise because of smooth operation and enlarge the duration of the devices such as the $\mathrm{ON} / \mathrm{OFF}$ valves $\mathrm{VS}_{2}, \mathrm{VS}_{3}$ and the hydraulic motor. Second, the steady state error in the working phase decreased dramatically in the PMT system, from 6 to 18 times, it is from 1.66 to $4.1 \mathrm{~min}^{-1}$ corresponding with the percentage errors of 0.28 to $0.59 \%$. Following, The energy consumption of PMT system reduced much, it was only from 4.8 to 7.7 times less than energy consumption in FST system.

However, PMT system remains some restrictions that need to be improved consist of long transient time in acceleration phase and taking time to tune the gain of PID controller.

\section{References}

[1] F L Yin, S L Nie, and J Ruan. Research on the reliability of sliding bearing support in a swash-plate type axial piston water hydraulic pump. Proc. of 2011 International Conference on Fluid Power and Mechatronics (FPM), 2011.

[2] B Hollingworth. The past, present and future of (water) hydraulics. Proc. of $12^{\text {th }}$ Scandinavian International Conference on Fluid Power, SICFP'11, 2011.

[3] P N Pham, K Ito, and S Ikeo. The application of simple adaptive control for simulated water hydraulic servo motor system. Proc. of International Conference on Industrial Technology IEEE ICIT, 2013.

[4] K Ito. Control performance comparison of simple adaptive control to water hydraulic servo cylinder system. Proc. of $19^{\text {th }}$ Mediterranean Conference on Control and Automation (MED), 2011.

[5] K Ito and S Ikeo. Robust velocity control of a water hydraulic servomotor system with parameter uncertainty. Proc. of SICE Annual Conference in Fukui, 2003.

[6] K Ito, H Takahashi, S Ikeo, and K Takahashi. Robust control of water hydraulic servo motor system using sliding mode control with disturbance observer. Proc. of SICE-ICASE International Joint Conference, 2006.

[7] P N Pham, K Ito, W Kobayashi, and S Ikeo. Analysis of velocity control performance and energy recovery efficiency of water hydraulic fluid switching transmission. Proc. of International Journal of Automation Technology, 6(4):457-467, 2012.

[8] M Linjama, M Huova, P Boström, A Laamanen, L Siivonen, L Morel, M Waldé, and M Vilenius. Design and implementation of energy saving digital hydraulic control system. Proc. of The Tenth Scandinavian International Conference on Fluid Power, SICFP'07, 2007.

[9] K Sanada. A study on hils of fluid switching transmission. Proc. of SICE-ICASE International Joint Conference, 2006.

[10] K Ito, W Kobayashi, P N Pham, and S Ikeo. Control and energy saving performance of water hydraulic fluid switching transmission. Proc. of $12^{\text {th }}$ Scandinavian International Conference on Fluid Power, SICFP'11, 2011.

[11] P N Pham, K Ito, W Kobayashi, and S Ikeo. Research on velocity error and energy recovery efficiency of water hydraulic fluid switching transmission. Proc. of $11^{\text {th }}$ International Conference on Automation Technology, 2011.

[12] S Habibi and A Goldenberg. Design of a new high performance electrohydraulic actuator. Proc. of 1999 IEEE/ASME International Conference on Advanced Intelligent Mechatronics, 1999.

[13] Y Lin, Y Shi, and R Burton. Modeling and robust discrete-time sliding-mode control design for a fluid power electrohydraulic actuator (eha) system. Proc. of IEEE/ASME Transaction on Mechatronics, 18(1):1-10, 2013.

[14] H W Beaty and J L Kirtley. Electric Motor Hand Book. McGRAW-HILL, 1998. 